\title{
Die Bedeutung der Familienpolitik in der gewerkschaftlichen Gleichstellungspolitik
}

Gute und engagierte Familienpolitik sei die beste Gleichstellungspolitik, so lautet ein neues Credo landauf, landab - auch auf der europäischen Ebene. Betrachtet man im Jahr der deutschen EU-Ratspräsidentschaft zunächst die europäische Ebene, so ist festzustellen, dass das Postulat der Gleichstellung der Geschlechter in Wirtschaft und Politik hier als ausgereiftes Politikfeld gilt. Gleichstellungspolitik ist für die Europäische Union ein Thema mit großer Tradition, das, so die deutsche Bundesregierung, „nachhaltig in den Strukturen und Beschlüssen verankert ist ". ${ }^{1}$ Gleichstellungspolitik galt der deutschen Ratspräsidentschaft als breit getragene Erfolgsstrategie. Sie wird aber auch als unerlässliches Instrument für eine erfolgreiche Familienpolitik betrachtet, und hier ist bereits der Perspektivenwechsel erkennbar, der sich in den letzten Wochen und Monaten verfestigte.

Auch Prof. Eberhard Eichenhofer kommt auf der Konferenz der deutschen Ratspräsidentschaft zum europäischen Sozialmodell zu dem Ergebnis, dass die Gleichstellung der Geschlechter ein altes Anliegen der europäischen Sozialpolitik sei, während sich die Familienpolitik als vergleichsweise neues Feld darstelle. Dabei bezieht er sich vor allen Dingen auf Art. 119 des EWGVertrages von 1957, in dem der Lohngleichheitsgrundsatz verankert ist, und er fragt in seinem Eingangsstatement danach, wie es denn 1957 bei Gründung der EWG um die Familie stand. „Sie lag damals noch ganz außerhalb des Gesichtskreises der Gemeinschaft. Denn eine Familie zu haben, galt als Privatsache, und sie berührte auch die damals noch von Männern dominierte Arbeitswelt scheinbar nicht. Familie mit Erwerbsarbeit miteinander in Einklang zu bringen, war aus der Sicht des Jahres 1957 noch keine allgemeine Zielvorstellung der
Sozialpolitik. Denn in vielen Mitgliedsstaaten der damaligen EWG war die Familienarbeit noch Sache der Frau und die Erwerbsarbeit die Domäne des Mannes“.2

Ebenso unbestritten ist sowohl im wissenschaftlichen als auch im politischen Kontext die Auffassung, dass die Europäische Gemeinschaft das Mandat zu einer aktiven und systematisch angelegten Gleichstellungspolitik habe. Es geht nicht nur darum, eine überwachende Rolle einzunehmen und die rechtliche Gleichstellung der Geschlechter durchzusetzen, sondern zu garantieren, dass die Gemeinschaft, aber auch die Nationalstaaten, eigenständige Regeln zur Herstellung der Gleichbehandlung entwickeln. Es geht darum, die Gleichstellung der Geschlechter auch tatsächlich durchzusetzen. Infolge der nach wie vor vorhandenen Benachteiligung von Frauen auf dem Arbeitsmarkt galt und gilt diesem Politikfeld besondere Aufmerksamkeit. Insbesondere die Europäischen Strukturfonds (ESF) legten frühzeitig die Grundlagen für zahlreiche Initiativen in der Regional- und Strukturpolitik für die modellhafte Entwicklung guter Praxis.

Bezüglich der Frage, welche Familienpolitik in Europa und in den Nationalstaaten entwickelt werden sollte, liegt die Sache insofern anders, da dieses Politikfeld erst seit etwas mehr als einem Jahrzehnt in den öffentlichen Diskurs gerät. Damit einhergehend erweitert sich der Blick auf das traditionell der Privatsphäre zugeordnete Familienleben und wird mit dem Ziel der Gleichstellung von Frauen und Männern in einem engen, ja unmittelbaren Zusammenhang gesehen. Das Verhältnis von nach außen gerichteter Arbeitswelt und nach innen gerichtetem Familienleben gerät in den Fokus der Realpolitik und somit der aktuellen politischen Auseinandersetzungen. Wie viel Privatheit verträgt das Berufs- leben? Und umgekehrt: Wie viel öffentliches Interesse und z. B. auch wie viel Professionalisierung benötigt die Familienpolitik? Als eine der zentralen Akteurinnen vertrat seit den 1970er Jahren in diesem Zusammenhang die politische Frauenbewegung die Auffassung: Alles Private ist politisch! Und mit dieser Grundüberzeugung verbunden ist die Thematisierung der patriarchalisch-kapitalistischen Trennung zwischen bezahlter Erwerbsarbeit und unbezahlter Haus- und Fürsorgearbeit. Gleiches gilt für die Bestrebungen und Forderungen, diese zu überwinden. ${ }^{3}$

\section{EUROPÄISCHE ZIELSETZUNGEN UND ANSÄTZE}

Seit mehreren Jahren verfolgt die LissabonStrategie erstmalig auch quantitative Ziele, um Gleichstellung und eine ausgewogenere Situation auf dem Arbeitsmarkt zu erreichen. Die Lissabon-Strategie sieht vor: Die Frauenerwerbsquote soll im europäischen Durchschnitt 60 \% erzielen. Hinzu

\footnotetext{
BMFSFJ (2007): Leitfaden Gleichstellungsstrategie als Erfolgsstrategie in der deutschen Ratspräsidentschaft, S. 2

2 Eichenhofer, E., (o.J.): Die Umsetzung des Europäischen Paktes für die Gleichstellung - Einführung, unveröffentlichtes Manuskript, S. 1

3 Notz, G. (2006): Warum flog die Tomate?, NeuUlm; Vogel, U.: (2007): Meilensteine der Frauen und Geschlechterforschung, Wiesbaden
}

Claudia Menne, Leiterin des Bereichs Gleichstellungs- und Frauenpolitik beim DGB Bundesvorstand. Arbeitsschwerpunkte: Gleichstellungspolitik, Sozial- und Arbeitsmarktpolitik

e-mail: Claudia.Menne@dgb.de 
kommt das Barcelona-Ziel einer 30 \%igen Kinderbetreuungsquote für Kinder unter drei Jahren. Ein Anliegen, dessen Umsetzung gerade in Deutschland aktuell sehr engagiert diskutiert wird.

Begleitet und ergänzt werden diese allgemein anerkannten und mit unterschiedlicher Intensität verfolgten Ziele durch einen im März 2006 verabschiedeten europäischen Pakt für Geschlechtergleichheit. Mit ihm sollen alle Bestrebungen zur Förderung der Gleichheit von Männern und Frauen in der Arbeitswelt unterstützt werden. Und dieser Pakt versteht sich auch als politisches Begleitinstrumentarium für den ebenfalls im März 2006 verabschiedeten EU-Fahrplan zur Förderung der Gleichstellung zwischen Männern und Frauen für die Jahre 2006 und $2010 .{ }^{4}$ Den europäischen und nationalen Sozialpartnern kommt in diesem Kontext eine große Bedeutung zu. Von ihnen wurde vor drei Jahren ein Aktionsrahmen verabschiedet, der derzeit auf nationaler Ebene unterschiedlich umgesetzt und angegangen wird.

Dieser europäische Aktionsrahmen definiert drei Handlungsfelder, die als gleichberechtigt verstanden werden. Folglich wird davon ausgegangen, dass sie nur im Zusammenwirken auch zu einer tatsächlichen Verbesserung hinsichtlich der Gleichstellung auf dem Arbeitsmarkt führen können. Die drei Handlungsfelder sind: die bessere Vereinbarkeit von Beruf und Familie, Entgeltgleichheit und bessere Karrierechancen für Frauen. ${ }^{5}$ Insbesondere der DGB vertritt die Auffassung, dass nur verschränkte Aktivitäten auf allen drei Handlungsfeldern einen echten Fortschritt bewirken können und dazu beitragen, bestehende Diskriminierungen aufzuheben.

\section{FAMILIENPOLITIK}

Die oben skizzierten Themenkomplexe erzeugen in Deutschland derzeit einen Strudel ideologischer Auseinandersetzungen und eine Wertediskussion, die tief auf dem deutschen Verständnis von Geschlechterrollen und Familienbild gründet. Der Siebte Familienbericht der Bundesregierung und zahlreiche aktuelle Studien stellen den Zusammenhang zwischen sich abzeichnendem demografischen Wandel und familienpolitischem Kurswechsel fest. Zum Kernthema bzw. Dreh- und Angelpunkt der Debatten gerät die Frage nach einer verbesserten Vereinbarkeit von Familie und Berufsleben. Erste Reformen sind in Deutsch- land nach zähem Ringen wirksam geworden. Herausragendes Beispiel ist das neue Elterngeld. Es entspricht einer langjährigen Forderung der Gewerkschaften. Jedoch bereits bei der Debatte um den Ausbau der Kinderbetreuungseinrichtungen leben alte ideologische Grabenkämpfe wieder auf. Dabei geht es im Grunde genommen doch eigentlich nur darum, die Barcelona-Ziele auch in Deutschland umzusetzen. Im europäischen Benchmark ist ganz klar, dass sich Deutschland in punkto Bewältigung des demografischen Wandels und Familienpolitik in etlichen Bereichen weit abgeschlagen auf einem der mittleren, wenn nicht sogar hinteren Plätze befindet.

Gelingt der Abschied vom alten Familienmodell mit Rollenstereotypen im politischen Diskurs, um sich damit der deutschen und europäischen Realität zu stellen? Wie sieht moderne Elternschaft aus? Wie gestaltet sich das Nebeneinander von bezahlter und unbezahlter Arbeit? Das sind Fragestellungen, die im 21. Jahrhundert neue Antworten verlangen.

Der Wandel familiärer Lebensformen und die sogenannte Modernisierung des Geschlechterverhältnisses sind bereits weit fortgeschritten und werden nicht nur mit den gewandelten Ansprüchen im Arbeitsleben und dem demografischen Wandel begründet, sondern auch und vor allem mit dem gestiegenen Bildungsniveau und der (gewünschten) steigenden Erwerbsbeteiligung von Frauen. Eine gleichstellungsorientierte Familienpolitik muss insbesondere Ideen und Impulse einbringen, die Männer dazu bewegt, stärker als bisher familienbedingte Auszeiten zu nehmen, sodass die Familienarbeit nicht einseitig Frauen an einer gleichberechtigten Partizipation am Arbeitsmarkt behindert.

Ebenso ist die Erkenntnis weit verbreitet, dass die Ära des Mannes, der einen Familienlohn allein erwirtschaften kann, auch vorüber ist. Die ökonomische Unabhängigkeit der Frau ist nicht nur ein legitimer Wunsch, sondern in vielen Fällen eine reale Notwendigkeit. In Zeiten der Globalisierung, in denen insbesondere der Druck auf die Löhne und Gehälter in Deutschland enorm ist und Reallöhne faktisch sinken, müssen und wollen Frauen diese entstehenden Lücken über ihre eigene Erwerbsarbeit kompensieren.

Für junge Frauen gilt, genauso wie für Männer, zunehmend das sogenannte ,adult worker"-Modell. Dieses Modell geht davon aus, dass alle Erwachsenen, die erwerbs- fähig sind, auch einer Erwerbsarbeit nachgehen. Ungeklärt ist bei diesem Modell, wie die Versorgungs-, Erziehungs- und Hausarbeit geleistet werden soll. In dieser Debatte, die in gewisser Hinsicht geschlechtsblind ist, wird die unbezahlte Haus- und Familienarbeit nach wie vor im Wesentlichen an die Frauen weiterverwiesen. Der Schwachpunkt des, ,adult worker“-Modells ist der alleinige Fokus auf die bezahlte Arbeit. Wichtig wäre es, den Blick zu erweitern und alle Formen unbezahlter Arbeit einzubeziehen. ${ }^{6}$

Damit stellt sich die Frage nach der neuen Aufteilung zwischen bezahlter und unbezahlter Arbeit aus Geschlechterperspektive. Und eine politische Antwort kann und muss eine stärkere Externalisierung von Fürsorgearbeit (Care Work) öffentlich thematisieren. Bezogen auf die Debatte um die Kinderbetreuung erleben wir in Deutschland gerade genau diese Auseinandersetzung. Auch bezüglich anderer Fragen, wie Bildung, aber auch Pflegeeinrichtungen, ist dieser Gedanke bereits in Ansätzen erkennbar und wird mit Sicherheit auch über die Große Koalition der Jahre 2006 bis 2009 hinaus weiterentwickelt werden müssen.

Der DGB hat sich bereits 2003 für einen Ausbau der Tageseinrichtungen für Kinder bis zum Schuleintrittsalter ausgesprochen und eindeutig die infrastrukturelle Förderung priorisiert. Diese Tageseinrichtungen müssen bedarfsgerecht, wohnortnah, pädagogisch qualifiziert sowie verlässlich und gleichzeitig flexibel sein. Und er forderte schon damals einen Rechtsanspruch für Kinder unter drei Jahren sowie eine schrittweise Senkung der Gebühren.7 Diese Forderungen wurden in der aktuellen Debatte bekräftigt.

\footnotetext{
4 Beiden gemeinsam sind Ziele, die darauf gerichtet sind, die Beschäftigung von Frauen in allen Bereichen des Arbeitslebens zu fördern, alle Formen der Diskriminierung zu bekämpfen, den Gleichheitsgrundsatz bei der Bezahlung weiterhin zu sichern und praktikabler zu gestalten sowie Vorurteile auf dem Arbeitsmarkt zurückzudrängen. Außerdem sei darüber nachzudenken, wie die Systeme der sozialen Sicherung für Frauen besser gestaltet werden können und wie Frauen im wirtschaftlichen und sozialen Leben mehr Einfluss erlangen können.

5 ETUC (2005): Business Europe; UEAPME CEEP (2005): Framework of Actions on Gender Equality.

6 DGB (2006): Frau geht vor, Info-Brief 3, S. $9 f$.

7 DGB Profil 03: Mehr als gute Gründe - Kindertageseinrichtungen schaffen Chancen.
} 


\section{DIE GRENZEN DER AKTUELLEN FAMILIENPOLITISCHEN DEBATTE}

Die aktuelle familienpolitische Debatte um neue Zielsetzungen und Fördermaßnahmen macht spätestens an den Fabriktoren und Bürotürmen halt, denn die Aufgabe, betriebliche Rahmenbedingungen zu schaffen, damit Männer und Frauen die Parallelität von Beruf und Familie auch leben können, gestaltet sich nach wie vor schwierig. Die steigende Verpflichtung von Unternehmen - auch in ihrem eigenen Interesse - einen gesellschaftspolitischen Beitrag $\mathrm{zu}$ leisten, ist eine der großen Aufgaben der derzeitigen Bundesministerin von der Leyen, die in vielen Gesprächen mit Unternehmensvertretern und Wirtschaftsverbänden für ihre Familienpolitik wirbt und dabei von Seiten des DGB und seiner Mitgliedsgewerkschaften ungeteilte Unterstützung erfährt. So beteiligen sich sowohl der DGB Vorsitzende Michael Sommer als auch der Vorsitzende der IG BCE, Hubertus Schmoldt, an der bundesweiten ,Allianz für die Familie“. Gewerkschaften entwickeln neue tariflich Forderungen zur besseren Vereinbarkeit, sie verbessern bestehende Regelungen und ergänzen sie um betriebliche Vereinbarungen. Weiterhin engagieren sich Betriebsräte und Gewerkschaften in Beratungsprojekten, die dabei unterstützen, Lösungen angepasst an die jeweilige betriebliche Situation zu etablieren.

Eine weitere Barriere ist gerade in den letzten Wochen ins öffentliche Bewusstsein gerückt unter dem Stichwort „Not am Mann“, womit die Wanderungsbewegungen innerhalb Deutschlands thematisiert werden. In mehreren Studien wurde belegt, dass junge Frauen in der Regel flexibler, veränderungswilliger und leistungsbereiter sind als junge Männer. Als Konsequenz daraus weisen mittlerweile weite Landstriche in den neuen Bundesländern einen deutlichen Mangel an jungen Frauen auf.
Sie sind vornehmlich in den Westen Deutschlands abgewandert, wo sie Arbeitsplätze und bessere Lebensbedingungen finden. Die Politik reagiert bisher relativ hilflos auf dieses Phänomen. Hier sind neben familienpolitischen vor allem arbeitsmarkt- und wirtschaftspolitische Instrumente gefordert, denn der Grad der öffentlich geförderten Kinderbetreuung liegt in den neuen Ländern traditionell über dem Bundesdurchschnitt.

\section{MÖGLICHE PERSPEKTIVEN}

Die gewerkschaftliche Frauen- und Gleichstellungspolitik plädiert im Einklang mit der EU dafür, dass die Vereinbarkeitsfrage nicht losgelöst von anderen ebenso wichtigen gleichstellungsrelevanten Faktoren diskutiert wird: die Frage nach dem Zugang zu wirtschaftlichen und politischen Ressourcen, das heißt die Herstellung der Entgeltgleichheit sowie die verstärkte Präsenz von Frauen in Entscheidungspositionen. Die DGB-Frauen vertreten nach wie vor die Auffassung, dass gesetzliche Regelungen hierfür auch für die Privatwirtschaft gelten sollten. ${ }^{8}$

In der politischen Lobbyarbeit setzen wir auf konkrete Praxisbezüge. Wir setzen uns dafür ein, dass betriebliche Lösungen zur Vereinbarkeit anstatt individuelle Lösungen gesucht werden, und dass Geschäftsführungen, Personalleitungen und Betriebsräte passgenaue beteiligungsorientierte Ansätze favorisieren. Nur so ist die notwendige Nachhaltigkeit gesichert. Die IG BCE setzt z. B. mit dem Chemie-Arbeitgeberverband auf den Abschluss von Betriebsvereinbarungen zur Familienfreundlichkeit, um der konkreten Umsetzung einen Rahmen zu geben, der Verlässlichkeit für beide Seiten beinhaltet.

Die IG Metall initiierte $z$. B. in den letzten Monaten ein Projekt im Bereich der Textilen Dienstleistungen und setzte das
Thema der Vereinbarkeit im IG Metall Bezirk Berlin-Brandenburg-Sachsen ganz oben auf die Tagesordnung.

In vielen dieser konkreten Fälle verknüpft sich in vielfältiger Weise Familienfreundlichkeit mit der Frage der Chancengleichheit zwischen den Geschlechtern. Nicht zuletzt das 2001 novellierte BetrVG bietet dafür verbesserte Initiativrechte. Öffentliche Projektförderung zur Schaffung guter Praxis wird auch auf lange Sicht ein entscheidender Beitrag sein, um die Instrumentenebene weiterzuentwickeln und Handlungsoptionen auszuprobieren. Der Weg zu einer flächendeckenden Veränderung des betrieblichen Alltags ist noch weit.

Bezüglich der beiden anderen genannten Barrieren ist zum einen der politische Dialog darüber dringend geboten, wie eine diskriminierungsfreie Arbeitswelt aussehen muss. Das neue AGG (Allgemeines Gleichstellungsgesetz) bietet hierfür viele Chancen. Der Antidiskriminierungsstelle des Bundes kommt nach Auffassung des DGB eine entscheidende Clearingfunktion bei dieser Aufgabe zu. Zum anderen sind unter dem Stichwort einer bevölkerungsbewussten Regionalpolitik (Bundesministerium für Verkehr, Bauen und Stadtentwicklung) neue strukturpolitische Ansätze zu konzipieren und ein offener Dialog zu führen, der auf monokausale Erklärungsmuster verzichtet. Die neue ESF99-Förderperiode 2007-2013 kann und sollte hierfür den geeigneten Rahmen bieten.

8 GB Bundesvorstand, Bereich Gleichstellungsund Frauenpolitik (Hrsg.) (2006): Gleich am Ziel?! Chancengleichheit in der Privatwirtschaft durch die freiwillige Vereinbarung und das AGG, Dokumentation einer gleichnamigen Fachtagung der FES und des DGB im September.

9 Europäischer Strukturfonds. 